\title{
Structural health monitoring with piezoelectric wafer active sensors - predictive modeling and simulation
}

\author{
Victor GIURGIUȚIU* \\ *Corresponding author \\ University of South Carolina, Department of Mechanical Engineering \\ 300 Main St. S., Columbia, SC 29208, USA \\ victorg@sc.edu
}

\begin{abstract}
This paper starts a review of the state of the art in structural health monitoring with piezoelectric wafer active sensors and follows with highlighting the limitations of the current approaches which are predominantly experimental. Subsequently, the paper examines the needs for developing a predictive modeling methodology that would allow to perform extensive parameter studies to determine the sensing method's sensitivity to damage and insensitivity to confounding factors such as environmental changes, vibrations, and structural manufacturing variability. The thesis is made that such a predictive methodology should be multi-scale and multi-domain, thus encompassing the modeling of structure, sensors, electronics, and power management. A few examples of preliminary work on such a structural sensing predictive methodology are given. The paper ends with conclusions and suggestions for further work
\end{abstract}

Key Words: structural health monitoring, SHM, nondestructive evaluation, NDE, piezoelectric wafer active sensors, PWAS, model-assisted probability of detection, MAPOD, hybrid global-local, HGL, finite element method, FEM

\section{INTRODUCTION}

Structural health monitoring (SHM) uses a set of permanently attached sensors to obtain on demand information about the structural performance and state of health [1].

The benefits of monitoring the structural state include design feedback, performance enhancement, on-demand condition-based maintenance, and predictive fleet-level prognosis. On-board structural sensing systems have been envisioned for determining the health of a structure by monitoring a set of sensors over time, assessing the remaining useful life from the recorded data and design information, and advising of the need for structural maintenance actions.

Piezoelectric wafer active sensors (PWAS) have emerged as one of the major SHM technologies; the same sensor installation can be used with a variety of damage detection methods: propagating ultrasonic guided waves, standing waves (E/M impedance) and phased arrays. Structural health monitoring (SHM) is a multidisciplinary process involving several disciplines that must be closely coordinated (Figure 1).

Guided-waves techniques for nondestructive evaluation (NDE) and structural health monitoring (SHM) applications are increasingly popular due to their ability to cover large areas with a relatively small number of sensors [2]. Miniaturized guided-wave transducers, such as piezoelectric wafers attached directly to structural elements, have gained large popularity due to their low cost, simplicity, and versatility [3]. These transducers can actively interrogate the structure using a variety of guided-wave methods such as pitch-catch, pulse-echo, phased arrays, and electromechanical (E/M) impedance technique. The can be also used passively for impact detection or acoustic emission (AE). These transducers can be 
developed into ultra-lightweight integrated ferroelectric thin films that may be manufactured directly on the structural materials through nano-fabrication techniques [4].

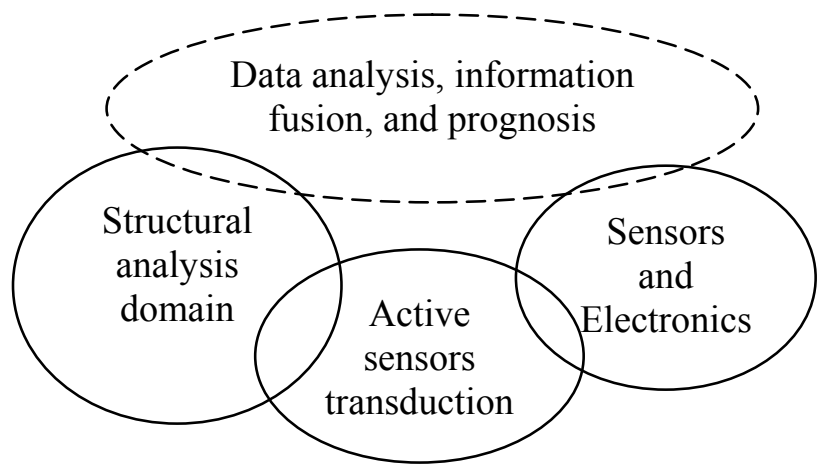

Figure 1 Venn diagram of the multi-domain interaction during structural sensing

An onboard SHM system could contain (a) sensors and sensor clusters; (b) electronics; (c) data processing and communications. The sensors can be either passive (strain, temperature, acceleration, etc.) or active (e.g., ultrasonic transducers that can interrogate the structure to detect damage presence, extent, and intensity). Passive structural sensing has been used to gather historical data about fleet usage and structural loads. Active structural sensing NDE techniques have been used to inspect the structure during maintenance actions, which are far apart and labor intensive. The desire exists for onboard active sensing systems that would interrogate the structure at will and produce on-demand structural health bulletins. The challenge in developing such active sensing systems is to develop integrated miniaturize transducers that can be permanently bonded to the structure and left in place to be activated on demand.

\section{PIEZOELECTRIC WAFER ACTIVE SENSORS}

Piezoelectric wafer active sensors (PWAS) couple the electrical and mechanical effects (mechanical strain, $S_{i j}$, mechanical stress, $T_{k l}$, electrical field, $E_{k}$, and electrical displacement, $\left.D_{j}\right)$ through the tensorial piezoelectric constitutive equations

$$
\begin{aligned}
& S_{i j}=s_{i j k l}^{E} T_{k l}+d_{k i j} E_{k} \\
& D_{j}=d_{j k l} T_{k l}+\varepsilon_{j k}^{T} E_{k}
\end{aligned}
$$

where, $s_{i j k l}^{E}$ is the mechanical compliance of the material measured at zero electric field $(E=0), \varepsilon_{j k}^{T}$ is the dielectric permittivity measured at zero mechanical stress $(T=0)$, and $d_{k i j}$ represents the piezoelectric coupling effect. PWAS utilize the $d_{31}$ coupling between in-plane strains, $S_{1}, S_{2}$, and transverse electric field, $E_{3}$. PWAS are transducers are different from conventional ultrasonic transducers because [5]:

1. PWAS are firmly coupled with the structure through an adhesive bonding, whereas conventional ultrasonic transducers are weakly coupled through gel, water, or air. 
2. PWAS are non-resonant devices that can be tuned selectively into several guidedwave modes, whereas conventional ultrasonic transducers are single-resonance devices.

3. Because PWAS are small, lightweight, and inexpensive they can be deployed in large quantities on the structure, which is not practical with conventional ultrasonic transducers, which are relatively bulky and expensive.

Propagating Lamb waves

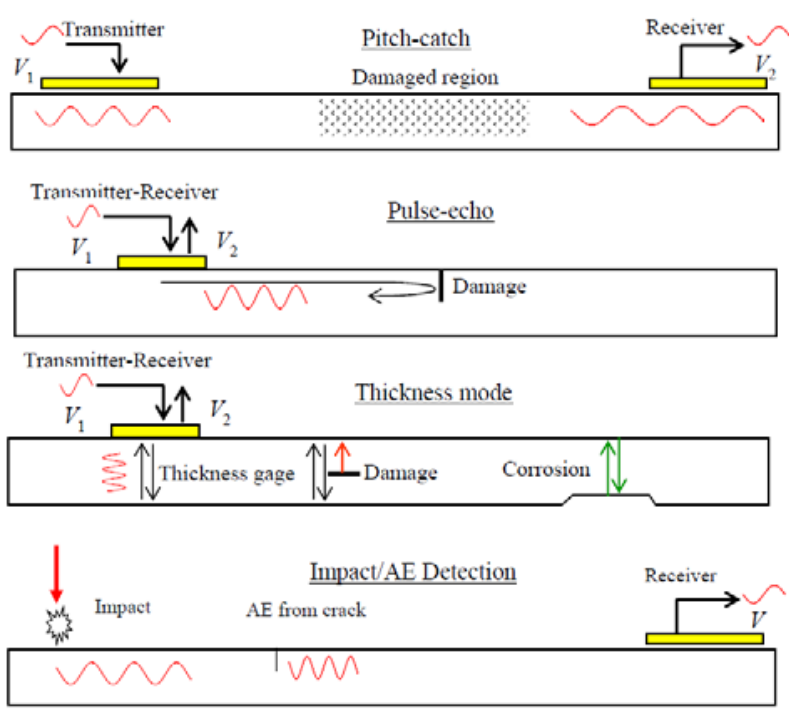

$\underline{\text { Standing Lamb waves }}$ (E/M Impedance)

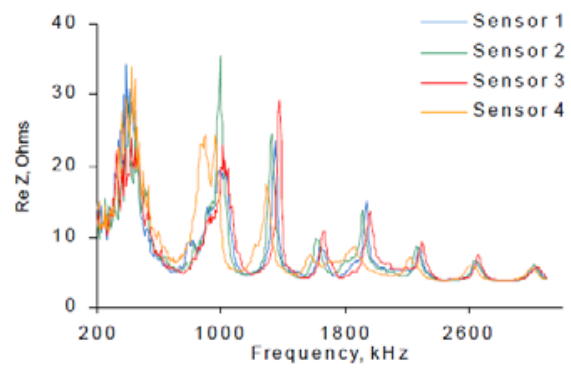

PWAS Phased Arrays

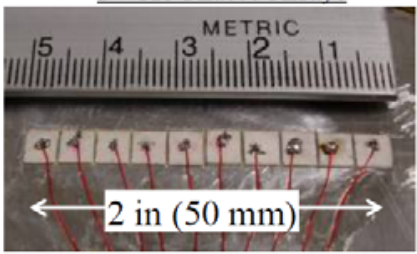

Figure 2 PWAS used for structural sensing include propagating Lamb waves, standing Lamb waves (electromechanical impedance) and phased arrays [5]

By using Lamb waves in a thin-wall structure, one can detect structural anomaly, i.e., cracks, corrosions, delaminations, and other damage.

PWAS transducers act as both transmitters and receivers of Lamb waves traveling through the structure. Upon excitation with an electric signal, the PWAS transmitter generates Lamb waves in a thin-wall structure. The generated Lamb waves travel through the structure and are reflected or diffracted by the structural boundaries, discontinuities, and damage. The reflected or diffracted waves arrive at the PWAS receiver where they are transformed into electric signals.

PWAS transducers can serve several purposes [5]: (a) high-bandwidth strain sensors; (b) high-bandwidth wave exciters and receivers; (c) resonators; (d) embedded modal sensors with the electromechanical (E/M) impedance method. By application types, PWAS transducers can be used for (i) active sensing of far-field damage using pulse-echo, pitchcatch, and phased-array methods, (ii) active sensing of near-field damage using highfrequency E/M impedance method and thickness-gage mode, and (iii) passive sensing of damage-generating events through detection of low-velocity impacts and acoustic emission at the tip of advancing cracks (Figure 2). The main advantage of PWAS over conventional ultrasonic probes is in their small size, lightweight, low profile, and small cost. In spite of their small size, PWAS are able to replicate many of the functions performed by conventional ultrasonic probes. 


\section{PREDICTIVE MODELING AND SIMULATION NEEDS FOR SHM APPLICATIONS}

The challenge in developing active sensing SHM systems is to develop integrated miniaturize transducers that can be permanently bonded to the structure and left in place to be activated on demand. Some of the technical gaps that hinder the implementation of onboard structural sensing systems are [6]:

- Sensing and recording systems have unproven reliability; high burden of data downloads; lack of validation analysis models from SHM data [7]

- Important effects are not accounted for: unstable boundary conditions; structural variability at different length scales; the need of a pristine-structure baseline; methods do not exist to avoid/account for environmental effects on structure [8]

- Validation and verification procedure are needed to assess probability of detection in the presence of structural, material, sensory, and algorithmic variability [8]

- Sensor performance may be affected by environmental exposure; the bond between the sensor and the structure is a critical durability issue that is not yet fully understood/controlled [9]

Embedded piezoelectric sensors have been used for in-flight structural health monitoring of bonded repairs and structural hot spots [10]. Besides laboratory experiments and flight tests, these studies have also attempted to model the sensor-structure assembly and to predict the sensor response to structural damage using finite element codes[11]. Current efforts are directed towards damage detection in composite structures [12].

Experimental studies have revealed that environmental effects can lead to the degradation of the ultrasonic guided-wave field created by an adhesively bonded piezoelectric wafer transducer. Accurate laser-based wave-field imaging results have shown that considerable wave-field distortion and amplitude degradation may occur due to environmental effects (e.g., temperature cycling). Attempts have been made to model the assembly of the piezoelectric wafer, underlying structure, and adhesive bonding layer using analytical models and specialized multiphysics finite element codes [9]. The need exists for a more profound understanding of such phenomena, which will allow us to recognize degradation of sensor effectiveness and compensate for it in data processing. Such understanding will also allow us to improve the design of sensor/structure adhesive interface in order to prevent sensor disbonding under extreme operational conditions.

SHM researchers have conducted a number of proof-of-concept experiments to demonstrate the feasibility of such an approach. Doyle, Zagrai, and Arritt [13] have used several active-sensing techniques for assessing bolted joint integrity such as the acoustoelastic phase change method. Other methods being considered are the electromechanical (E/M) impedance, pulse-echo, and nonlinear ultrasonics. These experimental studies have indicated the feasibility of using structural sensing for assessing the structural state and detecting flaws in certain cases. The methodology used in these studies has been to measure a set of pristine situations (training set) and use them as a baseline to identify changes in the signals that might be related to changes in the structural state. Though effective in many cases, such an approach (based entirely on experiments) may have significant implementation challenges, such as:

(a) Potential confusion between changes in structural configuration (modular component uncertainty, different component placement, various bolt patterns, etc.) and actual structural flaws (inadequate interface boundary conditions, delaminated panels, bolts not torqued correctly, etc.). 
(b) Difficulty of extending outside the training set when trying to accommodate new configurations needed for new missions, orbits, payloads, technology, etc.

A model-assisted strategy has been considered for transitioning the SHM principles from laboratories into actual systems [14]. The advantage of using a model-assisted approach is that parameter studies and carpet-plots can be performed computationally over a range of parameters and scenarios such that response trends can be detected. In particular, a modelassisted strategy could address the effect of structural and sensing variability on the probability of detection, which is still not as clear for SHM as it is for NDE. Essential for a model-assisted approach is its ability to perform "virtual sensing", i.e., to be able to predict the sensor response as function of structural changes, due to (a) damage or (b) changes in environmental and/or boundary conditions, and to confidently discriminate between (a) and (b). Such a capability does not exist, though it is highly desirable. It is apparent that the need exists for a predictive methodology that could perform simulation of various damage scenarios and predict the signals that would be generated by the SHM sensors.

A recent initiative in SHM technology development has been toward the development of wireless sensing in the desire to eliminate costly weight-adding cables [15]. Energy harvesting methods are being considered in order to have the wireless SHM system run autonomously for long periods of time without battery replacement [16]. Autonomous structurally monitoring systems are envisaged that should be able to (a) evaluate/quantify the health/damage state of the structure; (b) wirelessly communicate within the sensor network and with the outside user and (c) be self-sustained through energy harvesting. It is apparent that such a system needs to be modeled and designed in an integrated way with all its functionality properly optimized. In addition, some fundamental issues are still not clarified such as (a) how much energy can be harvested from the aircraft structural vibrations? (b) what should be done to avoid electromagnetic interference (EMI) between SHM wireless and the aircraft navigation and communication systems?

The need for a predictive modeling methodology that could relate directly the presence of structural flaws to changes in the signal of structural sensors is apparent. Such a predictive methodology would be able to simulate various structural scenarios (configuration changes, different flaws, temperature changes, etc.). A predictive modeling methodology would enable identifying the sensors sensitivity and specificity to structural changes (intentional or accidental), determining best sensor placement layout, running parameter studies, etc. To date, such a predictive modeling methodology does not exist, although some initial attempts have been made in simple cases by using finite element analysis [11], and model updating.

A major difficulty in developing a predictive modeling methodology for structural sensing is related to the multi-scale multi-domain aspects of the problem. The problem is multi-scale because it has to incorporate (a) the macro-scale structural features; (b) the micro-scale flaw/damage; (c) the mezo-scale interfaces between structural parts and between sensor and structure. The modeling is multi-domain because the analysis is integrated over several physical domains, i.e., (a) aerospace structural mechanics; (b) electromechanical transduction in the sensors; (c) guided wave ultrasonics; (d) power and signal electronics, etc. It is apparent that simply bolting together existing software codes (multiphysics finite elements, ultrasonics modeling, electronic circuit modeling) is not a credible option without an understanding of the interaction between the multi-domain variables native in each code and the multi-scale aspects of the problem. Fundamental studies are needed to clarify the multi-scale multi-domain interaction between structure, sensors, guided-wave patterns, damage progression, and signal processing and interpretation. In this way, one 
would achieve an understanding of how the hierarchical variables interplay, designation of what variables need to be passed between physical domains/codes, and an understanding of how multiple scales are to be addressed.

A coordinated effort is needed to address these fundamental research gaps and strive to develop an overall methodology for multi-scale multi-domain modeling of structural sensing. The methodology will be first validated on benchmark examples that are simple to understand but sufficiently representative to convey the concept. After the fundamental aspects are clarified, the work should address the analysis of actual structures by bringing together multiphysics finite element codes, semi-analytical finite element (SAFE) ultrasonic codes [17], electronic-circuits simulation codes, electric-power-management software, etc. One needs to interface various specialized software codes and make them work together in a synchronized assembly. The codes under consideration are:

Multiphysics finite element method (FEM) codes: most commercially available FEM codes have a multiphysics capability. In our preliminary work, we have experimented with ANSYS and ABAQUS multiphysics capabilities and obtained acceptable results. We were able to simulate both pitch-catch/pulse-echo wave propagation as well as E/M impedance standing waves using direct excitation of the piezoelectric wafer bonded to the structure. During these preliminary studies, we have also discovered some convergence differences between the two codes, whereas the same geometry analyzed with the same element type did not have same convergence characteristics in ABAQUS and ANSYS. Another widely used multiphysics code is COMSOL; we do not have first-hand experience with COMSOL, but we intend to acquire a license for this project in order to compare its performance with ABAQUS and ANSYS. The investigation of convergence behavior and development of convergence guidelines for multiphysics FEM simulation needs to be done.

Semi-analytical finite element (SAFE) approach has been previously developed for ultrasonics waveguides of complicated geometries, such as concrete reinforcing bars, tendons, track rails, 1-D stiffeners, etc. [17]. In the SAFE approach, the 1-D wave propagation along the waveguide is modeled analytically whereas the waveguide modes are found numerically through FEM discretization of the cross section. The SAFE approach can be used to model guided wave propagation in aerospace 1-D stiffeners such as stringers, ribs, frames, etc.

Electronic-circuits codes such as SPICE and its derivatives are used to simulate in realtime the steady state and transient behavior of active and passive electronic circuits. Rapid prototyping of electronic circuits with prescribed characteristics can be also attained. The SPICE codes should be used to model the behavior of the transmitter and receiver electronics for the piezoelectric transducers attached to the structure. Our aim is to optimize the electronics together with the transducers and the type of damage under consideration in order to achieve optimum detection capabilities.

Electric-power-management software exists that optimizes the PC power consumption towards achieving a green energy footprint. One needs to interface the electric power management software with the software codes described above such that minimum power consumption with maximum structural sensing capability will be achieved. The power optimization studies will be especially relevant for the future development of long-endurance SHM systems that can operated autonomously in stand-alone mode.

The focus of work should be on identifying the fundamental barriers in coupling/interfacing software from such diverse physical domains and disciplines. After the barriers are identified, a methodology for overcoming these barriers will be developed. 


\section{PRELIMINARY RESULTS IN SHM PREDICTIVE MODELING AND SIMULATION}

As already mentioned, the PI and his research group have accumulated extensive experience in the development of various active sensing methods using guided waves and PWAS transducers. Recent efforts have been focused on predictive modeling and some preliminary work has been performed as described next.
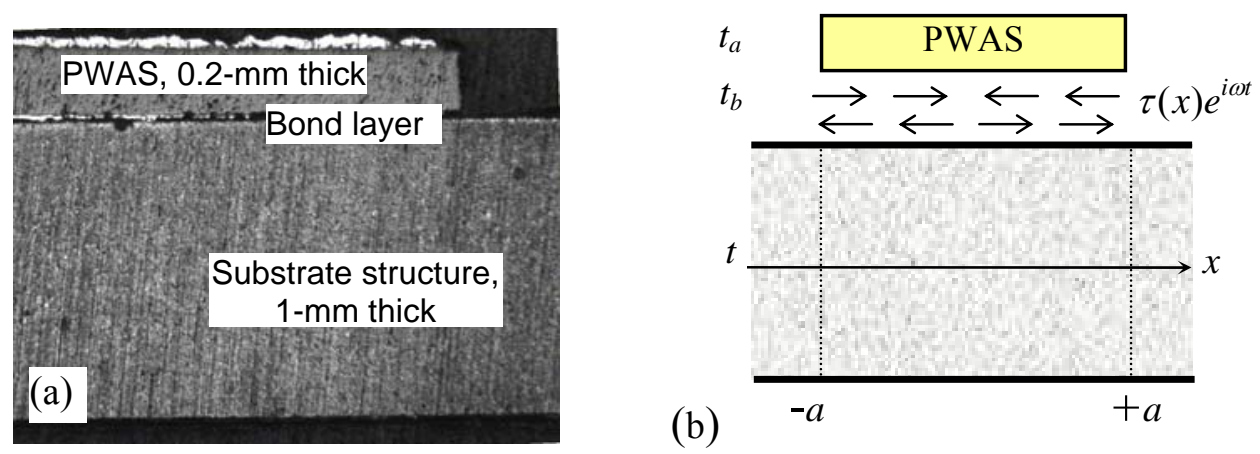

Figure 3 Bond-layer between PWAS and structure: (a) micrograph; (b) modeling [5]

\subsection{Shear-Lag Analysis of Structurally-attached PWAS}

Giurgiutiu and Santoni-Bottai [18] developed a shear lag solution for the stress/strain transfer between a structurally attached PWAS and the support structure (

Figure 3). Earlier studies of this subject assumed axial and flexural vibrations with linear strain distribution across the thickness; this assumption is fine for low values of the frequency-thickness product $f d$, but would not be appropriate for ultrasonic guided waves (e.g., Lamb waves) because the latter have complicated multi-mode strain distributions across the thickness. To overcome this limitation, Giurgiutiu and Santoni-Bottai [18] derived a generic shear lag solution that is not limited to the low frequency-thickness values.
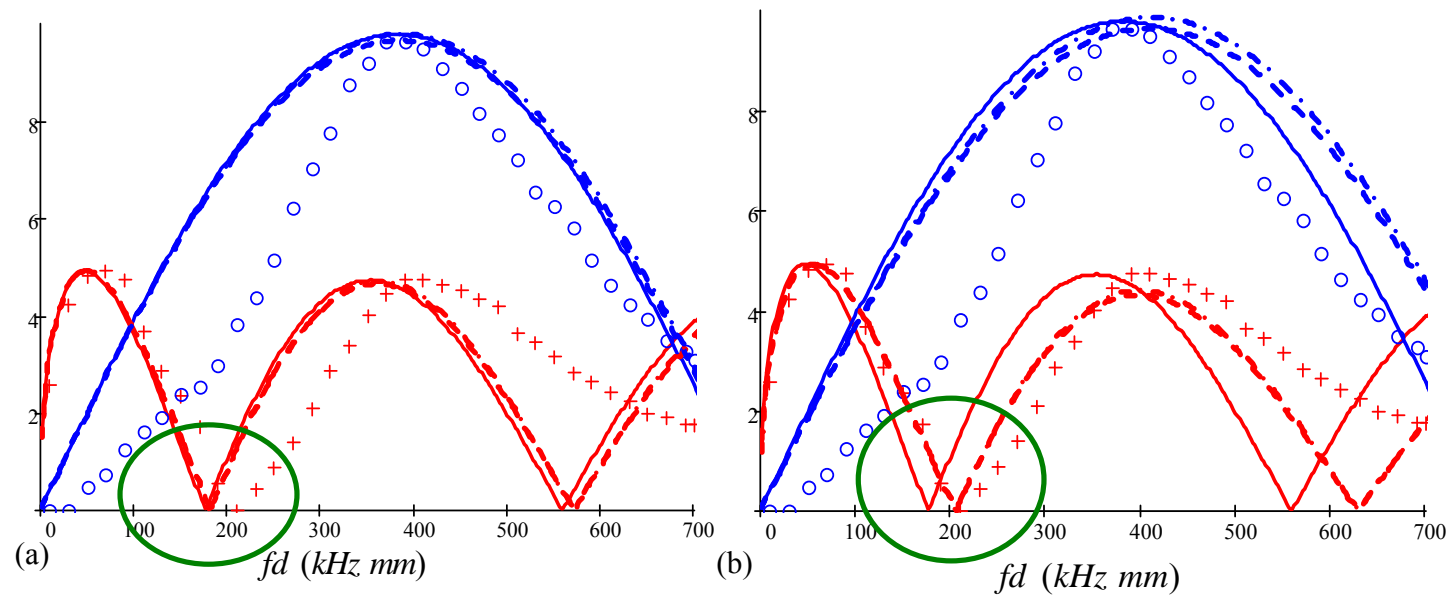

Figure $4 \quad$ PWAS tuning curves: (a) $t_{b}=1 \mu \mathrm{m}$; (b) $t_{b}=30 \mu \mathrm{m}$ [19]

This generic solution takes into account the exact thickness distribution of displacements and stresses corresponding to the Lamb wave modes existing at a particular 
ultrasonic frequency-thickness product value. This study [18] showed that essential parameters such as the axial-flexural repartition number, $\alpha$, and the shear lag parameter, $\Gamma$ as well as the tuning curves depend on the frequency-thickness product.

Santoni-Bottai and Giurgiutiu [19] extended this work to the case of multiple Lamb wave modes excited in the structure, the shear stress in the bonding layer depends on the number of modes present in the structure $M$, the PWAS size, $2 a$, the modal wavenumbers, $\xi_{m}, m=1, \ldots, M$, and the shear lag parameter $\Gamma$ and obtained an exact solution, which has shown a substantial improvement in the PWAS-Lamb wave tuning curves and an almost perfect match with the experimental measurements [19].

Figure 4a shows experimental and theoretical tuning curves for the first antisymmetric and symmetric modes on 1-mm aluminum plate. The amplitude of the theoretical curves have been scaled such as the first antisymmetric peak amplitude was the same as the experimental one. In Figure $4 \mathrm{a}\left(t_{b}=1 \mu \mathrm{m}\right)$, the maxima and the zeros of the antisymmetric theoretical curves are not in the same locations with the experimental ones, while the symmetric prediction curves are more close to the expected values. The prediction curves derived with improved theory are almost coincident for any frequency and they are closer to the solution through ideal bonding assumption at the low frequencies. In Figure $4 \mathrm{~b}$, the predicted curves are plotted for a thicker bond thickness $\left(t_{b}=30 \mu \mathrm{m}\right)$. The first antisymmetric maxima and minimum are now coincident with the experimental values, while the symmetric maxima have not changed their location significantly. As in Figure 4a, there is almost no difference between the predictions made for thicker bonds.

\subsection{Transfer Matrix Method Approach to Modeling the PWAS Detection of Disbonds}

Transfer matrix method (TMM) is an efficient analytical approach for solving 1-D vibration and wave propagation problems. TMM starts with the exact closed-form solutions describing the vibration and wave propagation in simple uniform segments and then uses a state-vector formulation and boundary matching to connect segments with different properties.

Cuc and Giurgiutiu [20] used the TMM approach to study adhesive bonds. The TMM approach permits the modeling of branched structures, such as in the case of a disbond or split in an adhesively bonded structure. The state vector at the right end of the beam is expressed in terms of the state vector at the left end of the beam using exact beam functions. Boundary conditions are matched at the left and right beam ends.

$$
\mathbf{z}_{B C}^{R}=\mathbf{P}_{2} \cdot \mathbf{z}_{B C}^{L}+\mathbf{P}_{1}^{F}=\mathbf{P}_{2} \cdot \mathbf{F}_{1} \cdot \mathbf{z}_{1}^{R}=\mathbf{P}_{2} \cdot \mathbf{F}_{1} \cdot\left(\mathbf{z}_{B C}^{L}+\mathbf{P}_{1}^{F}\right)
$$

Hence, one writes

$$
\begin{gathered}
\mathbf{z}_{B C}^{R}=\mathbf{U} \cdot \mathbf{z}_{B C}^{L}+\mathbf{T} \\
\mathbf{U}=\mathbf{P}_{2} \cdot \mathbf{F}_{1} \cdot \mathbf{P}_{1} \text { and } \mathbf{T}=\mathbf{P}_{2} \cdot \mathbf{F}_{1} \cdot \mathbf{P}_{1}^{F}+\mathbf{P}_{2}^{F}
\end{gathered}
$$

We have used the TMM approach to simulate analytically the detection of disbonds in adhesively bonded structures using PWAS transducers [20]. TMM was used for modeling a cracked multi-layer adhesively bonded beam with a PWAS attached to the top surface. The model starts with a single segment between points 1 and 2 . At point 2, the model splits into two branches representing the structure above and below the disbond. The upper branch is split into segments 2-3, 3-4, and 4-5 to accommodate the presence of the PWAS transducer between junctions 3 and 4 . The two branches reunite at junction 5 . The rest of the beam between 5 and 6 is modeled again with only one segment. 


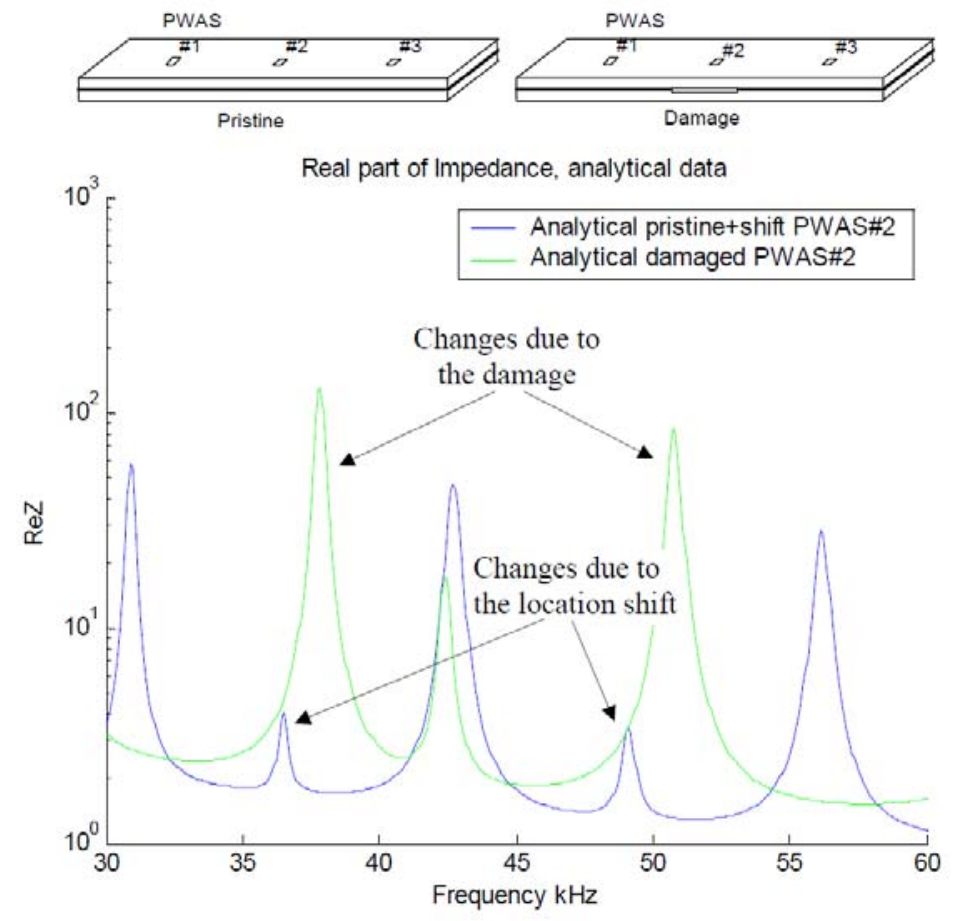

Figure 5 E/M impedance spectrum of a PWAS on adhesively bonded metallic coupon calculated with TMM [20]

Figure 5 shows the E/M impedance spectrum curves predicted for a small adhesively bonded coupon having three PWAS transducers mounted on the upper surface. This analytical study (which was backed up by experimental measurements) was aimed at determining two things:

(a) the changes that occur in the spectrum when disbonds appear in the adhesive joint

(b) the sensitivity of the spectrum to changes in the PWAS location

The spectrum shown in Figure 5 corresponds to PWAS \#2, which is placed directly above the disbond. Similar changes, though of lesser amplitude, were observed for PWAS \#1 and \#3, which are not placed on top of the disbond but in its vicinity. For the pristine beam, the E/M impedance spectrum of PWAS \#2 place exactly in the middle of the beam, shows three peaks in the $30-60 \mathrm{kHz}$ range, i.e., at $\sim 31 \mathrm{kHz}, \sim 43 \mathrm{kHz}$, and $\sim 56 \mathrm{kHz}$. When disbond damage was applied, these peaks shifted to $\sim 38 \mathrm{kHz}, \sim 42.5 \mathrm{kHz}$, and $\sim 52 \mathrm{kHz}$. The changes in the first and third peak are major, and easily detectable, as indicative of disbond damage presence. This answers point (a) above: it indicates that significant changes take place in the spectrum when disbond damage appears. To address point (b), we introduced a minute (1-mm) shift in the PWAS location. This 1-mm shift generated new small peaks at $\sim 36 \mathrm{kHz}, \sim 49 \mathrm{kHz}$, but left the three major peaks virtually unchanged. This answers point (b), i.e., it shows that the spectrum is also sensitive to changes in PWAS location; however, it shows that such changes are generally small, and do not impede our damage detection capability, because the changes due to disbond are of much larger amplitude. In addition, during the SHM process, the PWAS location is fixed, and hence the differences between the pristine baseline and the damaged spectra will only be due to damage. The effect of PWAS location is nonetheless important when trying to compare theoretical predictions and experimental results. 


\subsection{Power and Energy Transduction between PWAS and Structure}

An analytical investigation of power and energy transduction between PWAS and structure during the structural health monitoring process was recently performed by Lin and Giurgiutiu [21]. This preliminary work uses an analytical approach applied to the simple model depicted in Figure 6. The study used a 1-D analytical model to capture the power and energy flow from the electrical source energizing the transmitter PWAS through various stages of transduction up to the signal captured by an instrument connected to the receiver PWAS.

The model consists of a transmitter PWAS (A) and a receiver PWAS (B) bonded to a metallic beam. The following energy conversion stages were considered:

(a) piezoelectric transduction between source and transmitter PWAS

(b) mechanical transmission of shear stresses from the PWAS to the structure

(c) excitation of ultrasonic waves traveling through the structure from the transmitter to the receiver

(d) capturing of ultrasonic waves arriving at the receiver location

(e) mechanical conversion of structural waves into shear stresses acting from the structure onto the receiver PWAS

(f) piezoelectric conversion at the receiver PWAS and measurement by the electrical instrument.

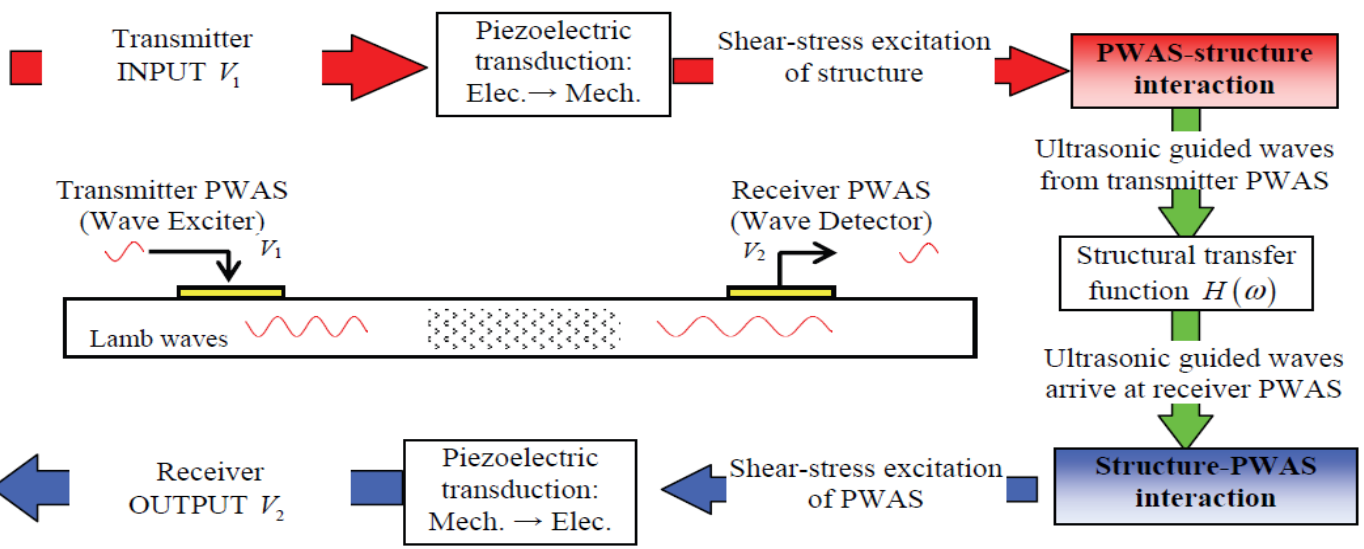

Figure 6 PWAS transmitter power and energy flow chart [21]

We have developed two analytical approaches, one based on standing waves (vibration), the other based on traveling waves. The standing-waves model is appropriate for a finitedimensions specimen; when excited harmonically, such a specimen will enter a state of vibration caused by the ultrasonic guided waves bouncing back and forth between the specimen boundaries in a standing-wave pattern. The traveling-waves model is appropriate for the study of large specimens in which the boundary effects can be neglected or for the study of wave-propagation events that happen before the waves bounce back from the reflecting boundaries. In order to account for the electronic circuit effects, considered a voltage source of voltage $V_{A}$, source impedance $Z_{A}$ and maximum current $I_{A \max }$ and measuring instrument characterized by instrument admittance $Y_{e}$. 
The standing-waves model is based on normal modes expansion; in the simplified case of only axial (extensional) and flexural (bending) vibrations, the voltage $V_{B}$ at $\mathrm{B}$ is found in terms of the voltage $V_{A}$ at $\mathrm{A}$ in the following form

$$
\begin{aligned}
\hat{V}_{B}(\omega)= & \frac{k_{31}^{2} Y_{0 B}}{Y_{e}+\left(1-k_{31}^{2}\right) Y_{0 B}} \times \\
& \frac{k_{i A} C_{A B}(\omega)}{R(\omega) k_{i A} k_{i B}\left[C_{A B}^{2}(\omega)-C_{A A}(\omega) C_{B B}(\omega)\right]+k_{i A} C_{A A}(\omega)+R(\omega) k_{i B} C_{B B}(\omega)-1} \hat{V}_{A}(\omega)
\end{aligned}
$$

where $Y_{0 B}$ is the admittance of PWAS B, $k_{i A}$ and $k_{i B}$ are the internal stiffnesses of PWAS $\mathrm{A}$ and $\mathrm{B}, k_{31}$ is the piezoelectric-transduction coupling factor of the PWAS material, The expressions $R(\omega), C_{A A}(\omega), C_{A B}(\omega), C_{B B}(\omega)$ are defined in ref. [21].

The propagating-waves model assumes that axial and flexural propagating waves generated at $\mathrm{A}$ are felt at $\mathrm{B}$ and transduced into an electrical voltage which, in turn, will produce a reflected wave that will be felt back at $A$ and will influence its ultrasonic output. Hence, the voltage $V_{B}$ at $\mathrm{B}$ is found in terms of the voltage $V_{A}$ at $\mathrm{A}$ in the following form

$$
\begin{aligned}
\hat{V}_{B}(\omega)= & \frac{k_{31}^{2} Y_{0 B}}{Y_{e}+\left(1-k_{31}^{2}\right) Y_{0 B}} \times \\
& \frac{k_{i A} C_{A B}(\omega)}{R(\omega) k_{i A} k_{i B} C_{A B}(\omega) C_{B A}(\omega)-\left(k_{i A} C_{A A}(\omega)-1\right)\left(R(\omega) k_{i B} C_{B B}(\omega)-1\right)} \hat{V}_{A}(\omega)
\end{aligned}
$$

The coefficients $C_{A A}(\omega), C_{A B}(\omega), C_{A B}(\omega), C_{B B}(\omega)$ are expressed in terms of propagating waves and are different than in previous equation (see ref. [21] for details). The model was used to predict the frequency response functions for voltage, current, complex power, active power, etc. At the input side, it was found that the reactive electric power is dominant and hence defines the size of the energizing power supply/amplifier (Figure 7a). At the PWAS structure interface, it was found that only the active electrical power gets converted into mechanical power, which is transmitted across the PWAS-structure interface and energizes the axial and flexural waves propagating into the structure. A parametric study was conducted w.r.t. the transmitter PWAS size: it was found that proper size and excitation frequency selection facilitates ultrasonic waves excitation through tuning effects. Figure 7 shows that a larger PWAS does not necessarily ensure more power transmission -careful frequency-size tuning is necessary! Similar tuning effects were also found at the receiver PWAS where a parametric study of the receiver size, receiver impedance and external electrical load provides useful design guidelines for PWAS-based sensing and/or energy harvesting.

one-Volt three-count smoothed tone burst was applied in all cases; the results, although similar, are not identical; this highlights the challenges that need to be overcome when performing such simulations. These models need to be first subjected to validation and verification with experiments and then extended to cover multi-modal Lamb waves, various structural situations (structural variability, structural joints, flaws/damage, nonlinear friction in joints and cracks, adhesive bonding/delamination, etc.), and more complicated excitation and detection electronic circuitry. 

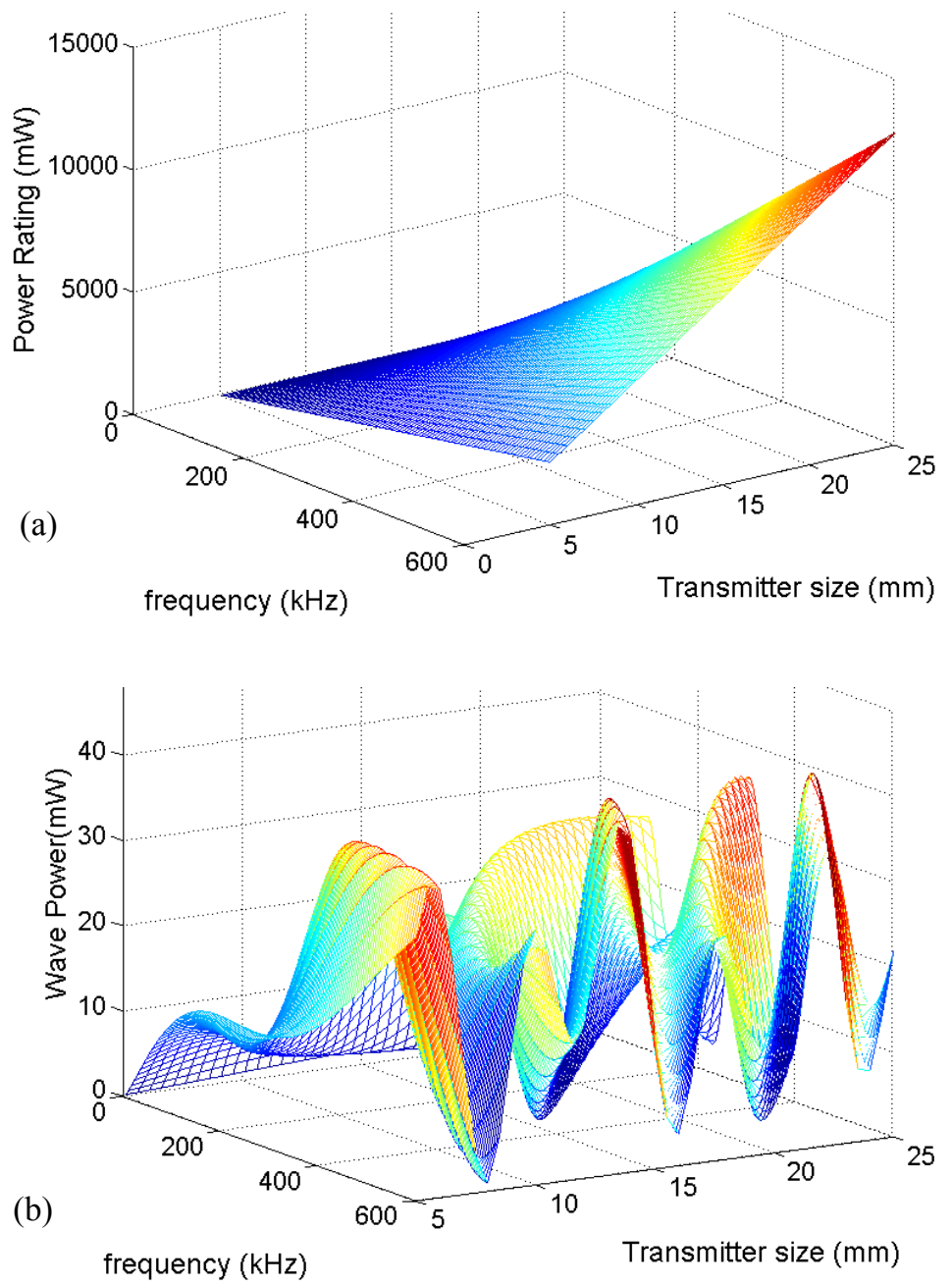

Figure 7 PWAS transmitter under constant 10-V excitation (a) power rating; (b) wave power[21]

\section{SUMMARY AND CONCLUSION}

This paper has started with a review of the state of the art in structural health monitoring with piezoelectric wafer active sensors and follows with highlighting the limitations of the current approaches which are predominantly experimental. Subsequently, the paper examined the needs for developing a predictive modeling methodology that would allow to perform extensive parameter studies to determine the sensing method's sensitivity to damage and insensitivity to confounding factors such as environmental changes, vibrations, and structural manufacturing variability. The thesis was made that such a predictive methodology should be multi-scale and multi-domain, thus encompassing the modeling of structure, sensors, electronics, and power management. The development of efficient hybrid globallocal finite element techniques was advocated and it was shown that the use of a modelassisted probability of detection approach is absolutely necessary. A few examples of preliminary work on such a structural sensing predictive methodology have been given. It is imperative that work on such a predictive methodology for structural sensing is started 
without delay in order to advance from an empirical approach into an analytical rational development of structural health monitoring systems and maintenance strategies.

\section{REFERENCES}

[1] White, E. V. (2009) "Structural Health Management: System Integration Challenges and Implications", ASME 2009 Conference on Smart Materials, Adaptive Structures, and Intelligent Systems (SMASIS 2009), Oxnard, CA, 21-23 Sept. 2009

[2] Thomson, D. O. and Chimenti, D. E. (Editors) (2008) Review of Progress in Quantitative Nondestructive Evaluation, Chapter 1B "Guided Waves", and Chapter 7A "New Techniques", AIP Conference Proceedings Vol. 28A, 2008

[3] Ihn, J.-B.; Chang, F.-K. (2008) "Pitch-catch Active Sensing Methods in Structural Health Monitoring for Aircraft Structures", Structural Health Monitoring - An International Journal, Vol. 7, No. 1, pp. 5-19

[4] Weaver, J.; Yuan, Z.; Liu, J.; Collins, G.; Chen, C. L.; Jiang, J. C.; He, J.; Meletis, E. I.; Guo, R. Y.; Bhalla, A.; Lin, B.; Giurgiutiu, V.; Cole, M. W. (2008) "Integration of Ferroelectric BaTiO3 Thin Films Directly on Ni and Ti Metallic Tapes for Structural Health Monitoring Systems and Energy Harvest Applications", Integrated Ferroelectrics, Vol. 100, pp. 61-71 (2008)

[5] Giurgiutiu, V. (2008) Structural Health Monitoring with Piezoelectric Wafer Active Sensors, Elsevier Academic Press, 760 pages, ISBN 978-0120887606, 2008

[6] Derriso, M. M.; Olson, S. E.; Desimio, M. P.; Pratt, D. M. (2007) "Why Are There Few Fielded SHM Systems for Aerospace Structures?", $6^{\text {th }}$ International Workshop on Structural Health Monitoring, Stanford Univ., CA, 11-13 Sept. 2007, pp. 44-55

[7] Stargel, D.; Tuegel, E. (2008) “Condition Based Maintenance Plus Structural Integrity”, AFOSR Structural Health Monitoring (SHM) Workshop, Covington, KY, 10 Aug. 2008

[8] Bunyak, C.; Lindgren, E. (2008) "SHM Verification and Validation Approach and Challenges", AFOSR Structural Health Monitoring (SHM) Workshop, Covington, KY, 10 Aug. 2008

[9] Blackshire, J. L.; Martin, S. A.; Na, J. K. (2007) "The Influence of Bond Material Type and Quality of Damage Detection for Surface Bonded Piezoelectric Sensors", $6^{\text {th }}$ International Workshop on Structural Health Monitoring, Stanford Univ., CA, 11-13 Sept. 2007, pp. 203-210

[10] Malkin, M.; Leonard, M.; Derriso, M. M.; Haugse, E. (2007) "Hot Spot Monitoring: Development of a Framework for SHM System Design", $6^{\text {th }}$ International Workshop on Structural Health Monitoring, Stanford Univ., CA, 11-13 Sept. 2007, 786-792

[11] Olson, S. E.; Desimio, N. P.; Derriso, M. M. (2006) "Analytical Modeling of Lamb Waves for Structural Health Monitoring”, $3^{\text {rd }}$ European Workshop on Structural Health Monitoring”, Granada, Spain, 5-7 July 2006, pp. 1070-1077

[12] Olson, S. E.; Desimio, N. P.; Brown, K. S.; Derriso, M. M. (2008) "Impact and Disbond Detection in Composite Wing Structure", 4th European Workshop on Structural Health Monitoring", Cracow, Poland, 2-4 July 2008, pp. 1271-1278

[13] Doyle, D., Zagrai, A., Arritt, B. J., Çakan, H., (2008) "Damage Detection in Satellite Bolted Joints," 2008 ASME SMASIS Conference on Smart Materials, Adaptive Structures and Intelligent Systems, Oct. 28-30, 2008, Ellicott City, Maryland, paper: SMASIS2008-550

[14] Lindgren, E. A.; Bunyak, C. F.; Aldrin, J. C.; Medina, E. A.; Derriso M. (2009) "Model-assisted Methods for Validation of Structural Health Monitoring Systems", 7th International Workshop on Structural Health Monitoring, Stanford Univ., CA, 9-11 Sept. 2009, pp. 2188-2195

[15] Lynch J P, Loh K. J. (2006) "A Summary Review of Wireless Sensors and Sensor Networks for Structural Health Monitoring", Shock and Vibration Digest, 38 91-128

[16] Park G., Rosing T., Todd M. D., Farrar C. R., Hodgkiss W. (2008) "Energy Harvesting for Structural Health Monitoring Sensor Networks", Journal of Infrastructure Systems, March 2008

[17] Bartoli, I.; Marzani, A.; Lanza di Scalea, F.; Rizzo, P.; Viola, E.; Sorrivi, E.; and Phillips, R. (2007) "SAFE Modeling of Waves for the Structural Health Monitoring of Prestressing Tendons," SPIE Vol. 6532, pp. 65320D1-65320D12, 2007

[18] Giurgiutiu, V.; Bottai-Santoni, G. (2009) “An Extension of the Shear Lag Solution for Structurally Attached 
Ultrasonic Active Sensors”, AIAA Journal, vol. 47, issue 8, pp. 1980-1983, Technical Notes, August 2009

[19] Santoni-Bottai, G.; Giurgiutiu, V. (2010) "Shear Lag Solution for Structurally Attached Active Sensors", SPIE International Symposium on Smart Structures and Materials + Nondestructive Evaluation and Health Monitoring, San Diego, CA, 7-11 Mar. 2010, paper \#7647-98

[20] Cuc, A.; Giurgiutiu, V. (2010) "Structural Health Monitoring of Adhesively Bonded Joints with Piezoelectric Wafer Active Sensors", Adhesion Science and Technology (in preparation)

[21] Lin, B.; Giurgiutiu, V. (2010) "Modeling Power and Energy Transduction of Embedded Piezoelectric Wafer Active Sensors for Structural Health Monitoring", SPIE International Symposium on Smart Structures and Materials + Nondestructive Evaluation and Health Monitoring, San Diego, CA, 7-11 Mar. 2010, paper \#7647-97 